\title{
ADEQUATE GINGIVAL RETRACTION AS PREREQUISITE FOR FABRICATION OF PRECISE FIXED PARTIAL DENTURES - ALGORITHM FOR CLINICAL IMPLEMENTATION
}

\author{
Iveta Katreva ${ }^{1}$, Maya Doychinova ${ }^{2}$ \\ ${ }^{1}$ Department of Prosthetic Dentistry, Faculty of Dental Medicine, \\ Medical University of Varna \\ ${ }^{2}$ Department of Conservative Dentistry and Oral Pathology, Faculty of Dental Medicine, \\ Medical University of Varna
}

\begin{abstract}
AIM: The aim of the paper is to introduce an algorithm for chemo-mechanical retraction with a-adrenomimetic decongestants as impregnating agents for adequate and safe gingival retraction - a prerequisite for production of precise fixed partial dentures (FPD).

MATERIALS AND METHODS: The clinical algorithm is based upon the results obtained from our previous researches and data gathered from the literature sources for effectiveness of $a$-adrenergic sympathicomimetic decongestants - $0.05 \%$ xylometazoline $\mathrm{HCl}, 0.05 \%$ tetrahydrozoline $\mathrm{HCl}$ and $10 \%$ phenylephrine $\mathrm{HCl}$, as alternative retraction agents.

Results: It is established that following the sequence of the suggested algorithm for the clinical use of $\alpha$-adrenomimetic decongestants provides sufficient degree of reversible horizontal and vertical gingival retraction without causing harmful effects to the periodontal tissue complex. The effect of conventional and alternative impregnating agents is compared, and the latter exhibits significantly better results both in penetration of the impression material and in terms of bio-tolerance towards the oral tissues. The working algorithm is presented with $0.05 \%$ xylometazoline $\mathrm{HCl}$, but it is universal for the whole chemical group of the researched compounds. It can be introduced in the routine dental practice with any a-adrenomimetic decongestant with no risk of adverse effects or mistakes.

CONCLUSION: The systematic algorithm favors the implementation of $\alpha$-adrenomimetic retraction agents in the clinical practice and helps to make the methodology popular among dental specialists.The application of $\alpha$-adrenomimetic decongestants is systemized within seven easy to follow procedures in order to obtain adequate gingival retraction - a mandatory condition in the fabrication of FPD.
\end{abstract}

Keywords: materials, biomaterials, gingival retraction, retraction agents, treatment protocol, fixed partial dentures

Address for correspondence:

Iveta Katreva

Faculty of Dental Medicine

Medical University of Varna

84 Tzar Osvoboditel Blvd

9000 Varna

e-mail: ikatreva@yahoo.com

Received: December 3, 2017

Accepted: December 29, 2017

\section{INTRODUCTION}

The rehabilitation of the mastication apparatus in modern prosthetic dentistry is related to the provision of periodontal health and prophylaxis of the oral cavity. The realization of a prosthetic treatment plan in accordance with the functional, prophylactic and esthetic indicators is directly related to the need for precise constructions, for which the quali-

Scripta Scientifica Medicinae Dentalis, 2017;3(2):13-17 
Adequate Gingival Retraction as Prerequisite for Fabrication of Precise Fixed Partial Dentures - Algorithm for Clinical ...

tative impression of the prosthetic field and in particular of the prepared teeth is decisive. Among the main criteria for high quality impression in modern clinical practice is the clear and distinct view of the gingival groove around the abutment teeth. This is the key for production of accurate fixed dental restorations without over-contouring and standing edges. The precision and excellent fit of the construction margins are of high prophylactic value as they prevent complications such as the appearance of secondary caries, inflammation of the adjacent periodontal tissues, detachment, etc. This is the only way to ensure a successful long-term treatment with fixed partial dentures (FPDs).

One of the main and frequently arising problems in impression making for fabrication of FPDs is to provide accessibility of the impression material not only to, but also beyond the preparation line. This is a critical moment for the precise reproduction of the contour details of the prepared abutment teeth on a gypsum working model in the dental laboratory $(1,2)$. The only possibility to overcome this problem is to have adequate gingival retraction preceding the impression, taking in the clinical protocol for FPD manufacturing.

In modern prosthodontics, direct optical impressions for FPD on natural teeth or implants have become increasingly popular in recent years as a result of the overall digitalization of dental medicine. It is a misconception that as a new technology, the digital impression "deals only" with the area below the gingiva. On the contrary, this methodology requires even wider (qualitative) temporary gingival displacement compared to conventional impression techniques (3). This fact proves that the question of methods and means of achieving effective gingival retraction remains relevant for future generations of dental practitioners.

The retraction of free gingival margin is a longestablished and well known technique that guarantees the exposure of the preparation's contours and makes visible the underlying unprepared hard tooth tissues allowing the penetration of the impression material into the gingival groove - conditions to which the high-quality final impression corresponds. M. Martignoni and A. Schönenberger find that a healthy marginal gingiva can easily be retracted by several tenths of a millimeter and the deformation induced lasts for a certain period of time in relation to the degree and duration of the pressure (4). This phenomenon makes the temporary displacement of tissues possible in two directions - vertically and horizontally. The manipulation provides not only ingress of the impression material into the gingival sulcus, but also a sufficient thickness of the layer, which is in direct relation with the dimensional stability of the impression and its tensile strength. Insufficient dilatation of the gingival groove leads to a poor quality of the final impressions and subsequently to low quality of produced FPD $(1,5)$.

Numerous materials and methods are described in the scientific literature for gingival retraction, but their choice depends on the clinical situation and often on the subjective choice of the dental medicine professional (6-9). The esthetic requirement for placing the preparation border inside the gingival sulcus is an obstacle for the impression taking and a challenge to gingival retraction techniques and devices. The most commonly applied method for gingival retraction is the chemo-mechanical one using retracing cords and chemical agents to control the bleeding and crevice fluid (10).

Some of the routinely used chemical substances, such as adrenaline, although applied topically, have an adverse effect on the whole organism and are contraindicated for use in patients with cardiovascular and some endocrine disorders. Other chemical substances cause inflammatory and caustic effects in prolonged contact with oral soft tissues. In search of the ideal chemical agent, researches revealed a new application for nasal and ophthalmic decongestants oxymetazoline hydrochloride, xylometazoline hydrochloride, tetrahydrozoline hydrochloride, which belong to the group of $\alpha$-adrenomimetic substances. It is proven that they lead to effective and reversible displacement of margo gingivalis without causing undesirable side effects on the oral and overall health of patients (11-13).

Therefore, the purpose of the article is to present an algorithm for routine clinical application of a-adrenomimetic substances and in particular $0.5 \%$ xylometazoline hydrochloride and $0.5 \%$ tetrahydrozoline hydrochloride as the most biocompatible alternative to conventional retraction agents. It will allow 
to obtain adequate displacement of the free gingival margin and to provide circumstances for accurate impressions and fabrication of FPD.

\section{MATERIALS AND METHODS}

An important advantage of the "new" chemomechanical agents for retraction over the conventional ones is the fact that they exhibit neither periodontal cytotoxicity, nor a damaging effect on hard tooth tissues. Promising data on the effective gingival retraction properties and the high bioavailability of $\alpha$-adrenomimetic impregnating substances are considered to be a sufficient argument for their popularization among the dental specialists.

Our previous experiments are related to another $\alpha$-adrenomimetic nasal decongestant $-0.05 \% x y$ lometazoline hydrochloride within the content of nasal drops Xylometazolin, Warsaw Pharmaceutical Works Polfa S.A., Poland. The analysis of the data shows higher efficiency of $0.05 \%$ xylometazoline $\mathrm{HCl}$ (Xylometazolin) in comparison to the ophthalmic decongestant $0.05 \%$ tetrahydrozoline $\mathrm{HCl}$ (Visine). Xylometazoline provides a thicker layer of impression material which penetrates deeper than half the gingival groove's depth (12-14). The results obtained from our previous researches, as well as the data gathered from the literature sources, demonstrate that $\alpha$-adrenergic sympathicomimetic decongestants - $0.05 \%$ xylometazoline $\mathrm{HCl}, 0.05 \%$ tetrahydrozoline $\mathrm{HCl}$ and $10 \%$ phenylephrine $\mathrm{HCl}$, achieve sufficient hemostasis, adequate reduction of the crevice fluid and effective retraction of the free gingiva, respectively.

Creating an algorithm for $\alpha$-adrenomimetic agents facilitates all dental practitioners and should make the method a preferred one. This approach allows the introduction of standards in conducting chemo-mechanical reversible dilatation of sulcus gingivalis, with predictable results and an objective assessment of the effect achieved.

In order to elaborate our algorithm, the nasal decongestant $0.05 \%$ xylometazoline $\mathrm{HCl}$ is selected from the group of $\alpha$-adrenomimetic substances for two reasons. It shows pronounced retraction effect, regardless of the impression material or the impression technique used. In over $55 \%$ of cases for A-silicon and over $76 \%$ for C-silicone, the results demonstrate penetration of the impression mass for more than half the depth of the gingival sulcus. The com- parative analysis of the results obtained with Xylometazolin and Visine categorically indicate that the first agent achieves a more effective free gingiva retraction by an average of $12-13 \%$ (13-15).

\section{RESULTS AND DISCUSSION}

A clinical algorithm for $0.05 \%$ xylometazoline $\mathrm{HCl}$ as a chemical agent for reversible displacement of free gingival margin is shown on Fig. 1. The algorithm for application of the nasal decongestant $x y l o-$ metazoline $\mathrm{HCl}$ as an impregnating agent for chemomechanical retraction contains 7 clinical steps.

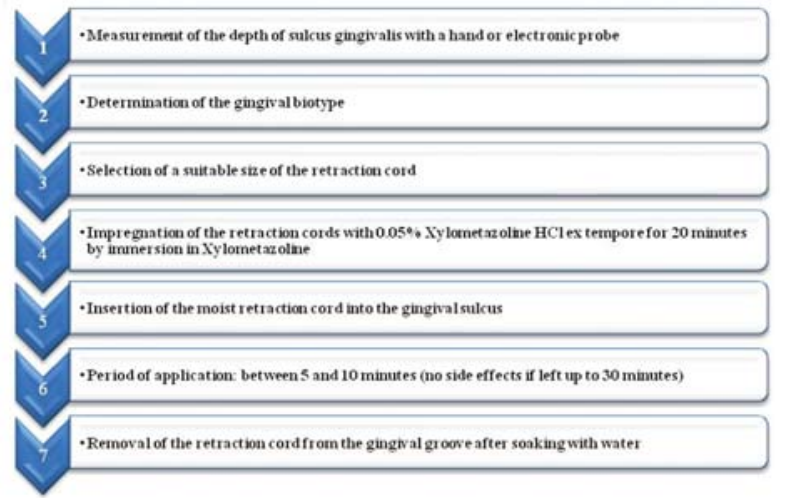

Fig. 1. Algorithm for clinical administration of $0.05 \% x y$ lometazoline $\mathrm{HCl}$ as impregnating agent for chemo-mechanical retraction of the gingival groove

It starts with initial measuring of the depth of sulcus gingivalis of the tooth/teeth subject to retraction with a manual or electronic periodontal probe. This information is necessary later for the third clinical step. Next, the gingival biotype is determined which should be taken in consideration when selecting the working approach with the retraction cord - "single" or "double cord" technique. On the third clinical step the suitable size retraction cord is selected depending on the previously measured depth of the gingival groove and the estimated biotype. Once the correct size of the cord is prepared, it is undergoing impregnation with $0.05 \%$ xylometazoline $\mathrm{HCl}$ ex tempore for 20 minutes by immersion in a commercially available nasal decongestant with this active ingredient at the indicated concentration $(0.05 \%$ Xylometazolin, Warsaw Pharmaceutical Works Polfa S.A., Poland) $(16,17)$. Insertion of the moist and slightly dried retraction cord into the gingival sulcus starts from the approximal area of the abutments 
Adequate Gingival Retraction as Prerequisite for Fabrication of Precise Fixed Partial Dentures - Algorithm for Clinical ...

with the help of a specially developed packer or any other non-traumatic suitable instrument (17-19). The retraction cord is correctly positioned when the circumferential outer contour of the preparation border is visible from occlusal view and the cord is not covered by a free gingiva. The period of application is between 5 and 10 minutes (17). The maximum possible stay of the retraction cords into sulcus gingivalis without any risk of complications caused by mechanical irritation is 30 minutes (20). The agent itself is non-cytotoxic and allows longer working time when it is necessary to provide a simultaneous retraction on multiple teeth. Prior to the removal of the cord from the gingival sulcus it should be soaked with water. This is a prophylactic measure to avoid possible hemorrhage recurrence despite the vasoconstrictor effect of the chemical agent.

The suggested algorithm can also be applied with the ophthalmic decongestant Visine $(0.05 \%$ tetrazolyne $\mathrm{HCl}$ ) without the risk of undesirable side effects. Following the sequence of the above-suggested algorithm for the clinical use of $\alpha$-adrenomimetic decongestants provides sufficient degree of reversible horizontal and vertical displacement of the free gingiva without causing harmful effects to the periodontal tissue complex. It should be mentioned that there are no subjective complaints from patients, as well as visible changes in the free and interdental gingiva in the postoperative period, which are typical after the use of astringents. These facts unambiguously prove the biocompatible behavior of $\alpha$-adrenomimetic decongestants as impregnating agents. They meet the requirement of the modern prosthetic treatment plan for providing periodontal health.

\section{CONCLUSION}

In this paper a systemic algorithm is developed for the clinical application of $\alpha$-adrenomimetic decongestants as alternative retraction agents for chemo-mechanical dilatation of the gingival groove. The retraction effect of conventional and alternative impregnating agents is compared, and the latter exhibit significantly better results both in penetration of the impression material and in terms of bio-tolerance to oral tissues.

The algorithm is developed for $0.05 \% x y$ lometazoline $\mathrm{HCl}$ as it is found to be most effective. Nevertheless it is universal for the group of a-adrenomimetic decongestants and can be applied in the same manner with any of these chemical agents for their excellent hemostasis without cytotoxic alternation of the periodontal tissues, harmful effect over hard tooth tissues and any risk for the overall health of the patients.

The use of these alternative retraction agents guarantees a prosthetic field in healthy state which directly correlates with the precision of the impressions and thus with the accuracy of the final FPD.

\section{REFERENCES}

1. Abdulaziz M. Gingival Retraction- Techniques and Materials: A Review. Pak Oral Dental J. 2010;30(2):3-14.

2. Gupta A, Prithviraj DR, Gupta D, Shruti P. Clinical Evaluation of Three New Gingival Retraction Systems:A Research Report. J Indian Prosthodont Soc. 2013;13(1):36-42. doi: 10.1007/ s13191-012-0140-y.

3. Al-Ani A, Bennani V, Chandler NP, Lyons KM, Tomson WM. New Zealand dentists use of gingival retraction techniques for fixed prosthodontics and implants. NZ Dent J. 2010;106:92-6.

4. Martignoni M, Schönenberger A. Precision fixed prosthodontics: clinical and laboratory aspects. Chicago: Quintessence; 1990. p.580.

5. Laufer BZ, Baharav H, Cardash HS. The linear accuracy of impressions and stone dies as affected by the thickness of the impression margin. Int J Prosthodont. 1994;73:247-52.

6. Shilingburg H, Hobo S, Whitsett L, Jacobi R, Bracket S. Fundamentals of Fixed Prosthodontics. Sofia: Quintessence Publishing Co, Inc; 2001. p. 260-77.

7. Donovan TE, Chee WW. Current concepts in gingival displacement. Dent Clin North Am. 2004;48(2):433-44. doi: 10.1016/j.cden.2003.12.012

8. Shaw DH, Krejci RF. Gingival retraction preference of dentists in general practice. Quintessence Int. 1986;17:277-80.

9. Blanchard JP. Nouvelle technique d'ouventure sulculaire pour l'empreinte en prothese fixee. Cah prothese. 2000;109:7-13.

10. Hansen PA, Tira DA, Barlow J. Current methods of finish-line exposure by practicing prosthodontists. J Prosthodont. 1999;8(3):163-70. 
11. Bowles WH, Tardy ST, Vaha-

di A. Evaluation of new gingival retrac-

tion agents. J Dent Res. 1991;70(11):1447-9. doi:

10.1177/00220345910700111101.

12. Katreva I, Abadjiev M, Simeonov S, Tivchev

O, Doychinova M, Tonchev TS. Application of

a-adrenomimetic decongestants as chemical agents for gingival retraction. Scr Sci Med Dent. 2015;2(1):17-23.

13. Katreva I, Georgieva K, Simeonov S, Doychinova M, Tonchev TS. Application of Xylometazoline for chemo-mechanical retraction of the free gingiva. J IMAB. 2015;21(3):849-52.

14. Katreva I, Abadzhiev M, Simeonov S, Tivchev O, Doychinova M, Bozukov HR, et al. Research of the attitude towards the application of alternative chemical agents for gingival retraction among the dentists. Varna Medical Forum. 2015;2(4):107-11.

15. Katreva I. Advantages of $\alpha$-adrenomimetic decongestants over conventional chemical agents for gingival retraction in order to avoid adverse systemic side effects - PhD thesis. Varna. 2015:145 (in Bulgarian).

16. Nowakowska $D$. The impact of retraction astringents on gingival margin tissues from literature review of in vivo studies. Protet. Stomatol. 2009;59(2):128-33.

17. Nowakowska D, Saczko J, Kulbacka J, Choromanska A, Raszewski Z. Cytotoxic potential of vasoconstrictor experimental gingival retraction agents -in vitro study on primary human gingival fibroblasts. Folia Biol (Praha). 2012;58(1):37-43.

18. Csempesz F, Vag J, Fazekas A. In vitro kinetic study of absorbency of retraction cords. J Prosthet Dent. 2003;89(1):45-9. doi: 10.1067/mpr.2003.61.

19. Rosenstiel SF, Land MF, Fujimoto J. Contemporary Fixed Prosthodontics. 4th ed. Mosby; 2006. p. 434-40.

20. Cloyd S, Puri S. Using the double-cord packing technique of tissue retraction for making crown impressions. Dent Today. 1999;18(1):54-9. 